\section{C6}

the magnitude of the ASE

effects is

modulated

by trans

genetic and environmental

factors that interact with the cis variants

$\Rightarrow$ GENE EXPRESSION

\title{
You are not alone - cis and trans factors both contribute to ASE
}

It has been proposed previously that differences in the expression of multiple alleles of a specific gene, which is known as allele-specific expression (ASE), may result from the direct effect of cis regulatory variants. Now, a twin study finds that trans genetic and environmental factors also play a part in ASE.

Buil et al. sequenced the mRNAs extracted from samples of fat, skin, blood and lymphoblastoid cell lines (LCLs) of 400 female twin pairs from the TwinsUK cohort and estimated ASE at every transcribed heterozygous single-nucleotide polymorphism (SNP) in each individual. To quantify the contribution of genetic and environmental factors to ASE, the researchers compared the estimated correlation of defined quantitative ASE phenotypes within monozygotic and dizygotic twin pairs. For dizygotic twins who shared $50 \%$ of the genome in the cis region, ASE phenotype correlation was less than half of that between monozygotic twins. This finding suggests the presence of non-additive genetic effects.

Further analyses found that nearly half of the heritability of ASE was due to a common cis expression quantitative trait locus (eQTL), indicating that although ASE arises mainly as a result of genetic variation in cis, it is also affected by genetic interactions and environmental factors. Indeed, $\sim 38-49 \%$ of the variance in the ASE observed was found to be due to gene-gene and gene-environment interactions.

Consistent with this result, several cases of gene-environment interactions were identified in fat tissues and LCLs. In particular, the expression of $A D I P O Q$ - which encodes the adipose tissue-derived hormone adiponectin, a protein known to be regulated by diet and exercise - was confirmed to depend on the interaction of a SNP with environmental factors.

The researchers propose a model in which ASE requires genetic variability in cis (that is, difference in the sequence of both alleles), but the magnitude of the ASE effects is modulated by trans genetic and environmental factors that interact with the cis variants.

Understanding the genetic architecture of gene expression is crucial to our understanding of how genetic variation affects complex traits. It will be interesting to investigate how such widespread gene-gene and geneenvironment interactions in ASE contribute to complex phenotypes, including diseases.

Esther Lau

ORIGINAL RESEARCH PAPER Buil, A. et al.

Gene-gene and gene-environment interactions detected by transcriptome sequence analysis in twins. Nature Genet. http://dx.doi.org/10.1038/ ng.3162 (2014)

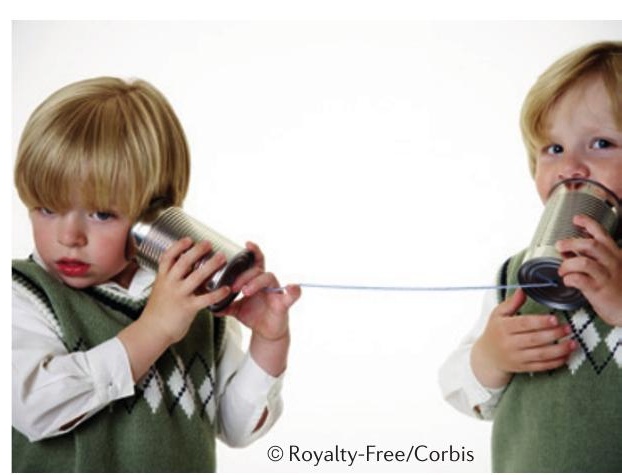

